\title{
KRAS Testing, Tumor Location, and Survival in Patients With Stage IV Colorectal Cancer: SEER 2010-2013
}

\author{
Mary E. Charlton, $\mathrm{PhD}^{\mathrm{a}}$; Amanda R. Kahl, $\mathrm{MPH}^{\mathrm{a}}$; Alissa A. Greenbaum, MD ; Jordan J. Karlitz, MD; \\ Chi Lin, MD, PhD ${ }^{\text {; }}$ Charles F. Lynch, MD, PhD; ; and Vivien W. Chen, PhD
}

\begin{abstract}
Purpose: KRAS mutations and tumor location have been associated with response to targeted therapy among patients with stage IV colorectal cancer (CRC) in various trials. This study performed the first population-based examination of associations between $K R A S$ mutations, tumor location, and survival, and assessed factors associated with documented KRAS testing. Methods: Patients with stage IV adenocarcinoma of the colon/rectum diagnosed from 2010 to 2013 were extracted from SEER data. Analyses of patient characteristics, KRAS testing, and tumor location were conducted using logistic regression. Cox proportional hazards models assessed relationships between KRAS mutations, tumor location, and risk of all-cause death. Results: Of 22,542 patients, $30 \%$ received $K R A S$ testing, and $44 \%$ of these had mutations. Those tested tended to be younger, married, and metropolitan area residents, and have private insurance or Medicare. Rates of KRAS testing also varied by registry (range, $20 \%-46 \%$ ). Patients with right-sided colon cancer (vs left-sided) tended to be older, female, and black; have mucinous, KRAS-mutant tumors; and have a greater risk of death (hazard ratio [HR], 1.27; 95\% Cl, 1.22-1.32). KRAS mutations were not associated with greater risk of death in the overall population; however, they were associated with greater risk of death among patients with left-sided colon cancer ( $\mathrm{HR}, 1.19 ; 95 \% \mathrm{Cl}, 1.05-1.33)$. Conclusions: This large population-based study showed that among patients initially diagnosed with stage IV CRC, right-sided colon cancer was associated with greater risk of death compared with left-sided cancer, and KRAS mutations were only associated with risk of death in left-sided colon cancer. An unexpected finding was that among patients with stage IV disease, right-sided cancer was more commonly seen in black patients versus whites. Future studies should further explore these associations and determine the role of biology versus treatment differences. In addition, use of $K R A S$ testing is increasing, but there is wide geographic variation wherein disparities related to insurance coverage and rurality may warrant further study.
\end{abstract}

Approximately 134,490 patients with colorectal cancer (CRC) were diagnosed in the United States in 2016. ${ }^{1}$ Of those, $21 \%$ had stage IV disease, with a 5-year relative survival of only $13.5 \% .^{2}$ Although a small proportion of these patients can be cured with surgery and neoadjuvant or adjuvant treatments,

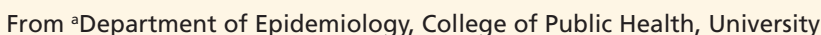
of lowa, lowa City, lowa; 'bepartment of Surgery, School of Medicine, University of New Mexico, Albuquerque, New Mexico; 'Division of Gastroenterology, School of Medicine, Tulane University, New Orleans, Louisiana; ${ }^{d}$ Department of Radiation Oncology, University of Nebraska Medical Center, Omaha, Nebraska; and 'Louisiana Tumor Registry and Epidemiology Program, School of Public Health, Louisiana State University Health Sciences Center, New Orleans, Louisiana.

Submitted March 3, 2017; accepted for publication July 12, 2017.

The authors have disclosed that they have no financial interests, most are incurable, and treatment focuses on prolonging life and improving quality of life. Targeted agents such as epidermal growth factor receptor (EGFR) inhibitors, including cetuximab, can inhibit tumor growth, but studies have shown that tumors with mutations in the KRAS gene (KRAS-mutant) respond poorly to anti-EGFR therapy (ClinicalTrials.gov arrangements, affiliations, or commercial interests with the manufacturers of any products discussed in this article or their competitors.

This work was supported in part under $\mathrm{NIH} / \mathrm{NCl}$ contract number HHSN261201000030C with Louisiana State University Health Sciences Center (V.W.C.); NIH/NCI contract number HHSN261201300020I with University of lowa (M.E.C., A.R.K., C.F.L.).

Correspondence: Mary E. Charlton, PhD, Department of Epidemiology, University of lowa College of Public Health, 145 North Riverside Drive, Room S453 CPHB, lowa City, IA 52242. E-mail: mary-charlton@uiowa.edu 
KRAS Status and Tumor Location in Stage IV CRC

identifiers: NCT00113763, NCT00113776). ${ }^{3-9}$ In 2009, NCCN recommended KRAS testing for patients with stage IV CRC at the time of diagnosis, and that only patients with tumors harboring the wild-type KRAS gene (KRAS-WT) be treated with anti-EGFR therapy. ${ }^{10}$ Subsequently, KRAS testing was included as a CRC site-specific factor (SSF 9) to be collected by NCI SEER registries, beginning with patients diagnosed in 2010, as part of the Collaborative Stage (CS) Data Collection System, version 2.

In our previous study of the SEER KRAS variable, ${ }^{11}$ only $23 \%$ of patients diagnosed in 2010 with stage IV CRC were KRAS tested, and there was variation in testing rates among registries. Of those tested, no survival differences were seen between the $40 \%$ of patients with KRAS-mutant tumors and the $60 \%$ of those with KRAS-WT tumors. This was unexpected because patients with KRAS-WT tumors could receive anti-EGFR to lengthen survival, but only 1 year of follow-up was assessed.

Recent studies suggest KRAS mutation status is not the only factor that should be considered when selecting chemotherapeutic interventions in stage IV CRC. CRC is a molecularly heterogeneous entity, resulting in differing mechanisms of carcinogenesis between sporadic right-sided colon cancers and left-sided colon cancers. ${ }^{12,13}$ It has previously been shown that right-sided cancer is associated with older age ( $\geq 75$ years), female sex, larger tumor size, higher tumor grade, ${ }^{14}$ and more frequent mucinous subtypes, ${ }^{15}$ as well as microsatellite instability (MSI), ${ }^{16,17}$ BRAF mutation, ${ }^{18,19}$ EGFR expression, ${ }^{20}$ KRAS mutation, ${ }^{20,21}$ and PIK3CA (Phosphatidylinositol 3 kinase, catalytic, alpha polypeptide) mutation. ${ }^{22}$ They are also more frequently associated with advanced disease, poorly differentiated tumors, and mucinous histology compared with left-sided cancers. ${ }^{18}$ Left-sided cancers demonstrate increased expression of EGFR and higher frequency of chromosomal instability. ${ }^{18,23,24}$ Clinical outcomes based on tumor location alone have been debated, although many trials have shown KRAS-WT left-sided cancers may have better overall and progression-free survival and an improved response to anti-EGFR therapy compared with right-sided cancers. Two trials have demonstrated that patients with KRAS-WT left-sided cancers survived longer if their treatment included cetuximab, whereas those with KRAS-WT right-sided cancers survived longer if their treatment included bevacizumab (antibody against VEGF-A) (ClinicalTrials.gov identifier: NCT00265850). ${ }^{25}$

In light of these recent studies highlighting the importance of sidedness on survival, our primary objective was to examine both KRAS status and sidedness in patients with stage IV CRC using population-based SEER data. Our second objective was to assess trends and factors associated with KRAS testing captured by SEER registries.

\section{Methods}

\section{Patients}

SEER*Stat version 8.3.2 was used to extract microscopically confirmed stage IV adenocarcinomas of the colon or rectum diagnosed in 2010-2013 from SEER registries with at least 100 patients with CRC. ${ }^{26}$ These registries included California, Georgia, Connecticut, Detroit, Hawaii, Iowa, New Mexico, Seattle-Puget Sound, Utah, Kentucky, Louisiana, and New Jersey. Patients were excluded if diagnosed on autopsy or death certificate only and had a nonspecific ICD-O-3 site code (ie, colon, not otherwise specified [NOS]). Access to SSF 9 (KRAS status) was granted by the NCI. SEER guidelines for coding SSF 9 stated that registrars should use all information gathered through completion of surgery in the first course of treatment, or all information available within 4 months of the diagnosis date in the absence of disease progression, whichever is longer. ${ }^{27}$ The Human Subjects Office did not consider this study to be human subjects' research.

\section{Study Variables}

Outcome variables included documentation of KRAS testing, KRAS results, and survival as of December 31, 2013. Testing was considered performed if SSF 9 contained values of "abnormal (mutated)" or "normal (wild-type)"; otherwise KRAS testing was considered not performed if SSF 9 values contained "test ordered, results not in chart," "not done," or "unknown."

Patient demographic variables at time of diagnosis included age, registry, sex, race/ethnicity, diagnosis year, insurance status, marital status, and residence in metropolitan versus nonmetropolitan/rural area. Tumor characteristics included location (rightsided $=$ cecum, ascending colon, hepatic flexure, 
transverse; vs left-sided = splenic flexure, descending colon, sigmoid, rectosigmoid junction); histology (adenocarcinoma vs mucinous adenocarcinoma); grade; $\mathrm{T}, \mathrm{N}$, and $\mathrm{M}$ stages, which was further broken down by metastatic site when sample size allowed; and single cancer versus multiple cancers (separate primary cancer before, during, or after diagnosis of stage IV CRC). Treatment variables included surgery and radiation therapy (yes/no).

\section{Statistical Analysis}

Logistic regression was used to determine characteristics associated with receipt of KRAS testing and with right-sided cancer (vs left). All variables listed previously were considered for inclusion in the KRAS testing model, and age, sex, race, diagnosis year, grade, TNM stage, and single versus multiple cancers were considered for inclusion into the right-sided CRC model because of their potential plausible relationship to tumor sidedness. Four Cox proportional hazards models were constructed to evaluate associations between KRAS testing, tumor location, and survival while controlling for patient, tumor, and treatment characteristics: (1) patients with CRC regardless of KRAS testing status or tumor site (left vs right vs rectum); (2) patients with CRC who received KRAS testing; (3) patients with left-sided cancer who received KRAS testing; and (4) patients with right-sided cancer who received KRAS testing. Only patients diagnosed in 20102012 were included in survival analyses to allow for sufficient follow-up. The following variables were included in all survival models regardless of significance: KRAS results, age, race, diagnosis year, insurance, marital status, stage group, $\mathrm{T}$ stage, $\mathrm{N}$ stage, and $\mathrm{M}$ stage/metastatic sites. Analyses were conducted using SAS 9.4 (SAS Institute, Cary, $\mathrm{NC})$.

\section{Results}

There were 27,231 patients with stage IV CRC identified and 22,542 (83\%) met inclusion criteria (2,598 were excluded because tumor site was nonspecific; 29 were death certificate-only; 1,272 were not microscopically confirmed; 731 had nonadenocarcinoma histology; and 59 were from Alaska). The overall KRAS testing rate was 30\% (Table 1). Of the

\begin{tabular}{|c|c|c|c|}
\hline & $\begin{array}{c}\text { Total } \\
(\mathrm{N}=22,542) \\
\mathrm{n}(\%)\end{array}$ & $\begin{array}{c}\text { Colon } \\
(\mathrm{N}=15,843) \\
\mathrm{n}(\%)\end{array}$ & $\begin{array}{c}\text { Rectal } \\
(\mathrm{N}=6,699) \\
n(\%)\end{array}$ \\
\hline \multicolumn{4}{|l|}{ KRAS values } \\
\hline $\begin{array}{l}\text { 010: Abnormal } \\
\text { (mutated) }\end{array}$ & 3,005 (13.3) & $2,215(14.0)$ & $790(11.8)$ \\
\hline 020: Normal (wild-type) & $3,789(16.8)$ & $2,682(16.9)$ & $1,107(16.5)$ \\
\hline $\begin{array}{l}\text { 997: Test ordered, } \\
\text { results not in chart }\end{array}$ & $268(1.2)$ & $200(1.3)$ & $68(1.0)$ \\
\hline 998: Test not done & $8,125(36.0)$ & 5,689 (35.9) & $2,436(36.4)$ \\
\hline 999: Unknown & 7,355 (32.6) & 5,057 (31.9) & $2,298(34.3)$ \\
\hline \multicolumn{4}{|l|}{ KRAS tested } \\
\hline Yes & $6,794(30.1)$ & 4,897 (30.9) & $1,897(28.3)$ \\
\hline No & $15,748(69.9)$ & $10,946(69.1)$ & $4,802(71.7)$ \\
\hline
\end{tabular}

6,794 tested, 44\% were KRAS-mutant and 56\% were KRAS-WT.

\section{KRAS Testing}

Table 2 displays frequencies and row percentages of patient/tumor characteristics by KRAS testing status, as well as odds ratios (ORs) and 95\% confidence intervals from a logistic model in which the dependent variable was KRAS testing and the independent variables included all those listed. Univariate analysis demonstrated that KRAS testing rates most substantially varied by age ( $43 \%$ for those $<30$ years vs $15 \%$ for $\geq 80$ years), registry ( $46 \%$ in Seattle-Puget Sound vs $20 \%$ in Louisiana), diagnosis year (25\% in 2010 vs $35 \%$ in 2013), and marital status (33\% for married vs $21 \%$ for widowed). The following variables were significantly associated with KRAS testing in the logistic model $(P<.05)$ : younger age, registry (California was referent because it had the largest number of patients), later year of diagnosis, covered by private insurance or Medicare (vs Medicaid or uninsured), married, metropolitan area residence, colon cancer (vs rectal), well-differentiated grade (vs unknown), $\mathrm{N}$ stage $\geq \mathrm{N} 1 \mathrm{a}$, and metastasis to liver only, lung only, or multiple organs (vs other single organ/site or metastases NOS) (Table 2).

KRAS testing variation by registry and diagnosis year were examined and results are provided in Figure 1. Rates increased each year among most registries; Connecticut experienced the largest increase (103\%), with $23 \%$ tested in 2010 and $46 \%$ in 2013 , followed by Iowa (95\%), with 21\% tested in 2010 and $41 \%$ in 2013. A few registries showed some decreases in testing between years, including Utah, Hawaii, and Kentucky. 
KRAS Status and Tumor Location in Stage IV CRC

\begin{tabular}{|c|c|c|c|}
\hline & $\begin{array}{c}\text { No KRAS Testing } \\
(\mathrm{N}=15,748) \\
\mathrm{N}(\%)\end{array}$ & $\begin{array}{c}\text { KRAS Testing } \\
(\mathrm{N}=6,794) \\
\mathrm{N}(\%)\end{array}$ & $\begin{array}{c}\text { aOR }^{\mathrm{a}} \\
(95 \% \mathrm{Cl})\end{array}$ \\
\hline \multicolumn{4}{|l|}{ Age, y } \\
\hline $0-29$ & $104(57.5)$ & $77(42.5)$ & $4.96(3.59-6.84)$ \\
\hline $30-39$ & $360(52.3)$ & $329(47.8)$ & $5.26(4.37-6.34)$ \\
\hline $40-49$ & $1,448(59.3)$ & $996(40.8)$ & $3.93(3.44-4.49)$ \\
\hline $50-59$ & $3,211(64.5)$ & $1,766(35.5)$ & $3.20(2.84-3.61)$ \\
\hline $60-69$ & $4,053(68.6)$ & $1,854(31.4)$ & $2.58(2.31-2.89)$ \\
\hline $70-79$ & $3,380(73.9)$ & $1,196(26.1)$ & $1.94(1.73-2.18)$ \\
\hline$\geq 80$ & $3,192(84.7)$ & $576(15.3)$ & 1.00 [Ref] \\
\hline \multicolumn{4}{|l|}{ Sex } \\
\hline Female & $7,144(70.6)$ & $2,972(29.4)$ & $0.99(0.93-1.06)$ \\
\hline Male & $8,604(69.2)$ & $3,822(30.8)$ & 1.00 [Ref] \\
\hline \multicolumn{4}{|l|}{ Registry } \\
\hline California & $6,041(71.0)$ & $2,473(29.1)$ & 1.00 [Ref] \\
\hline Connecticut & $630(61.3)$ & $397(38.7)$ & $1.64(1.42-1.88)$ \\
\hline Detroit & $848(67.0)$ & $417(33.0)$ & $1.23(1.07-1.40)$ \\
\hline Georgia & $1,788(67.8)$ & $848(32.2)$ & $1.11(1.00-1.23)$ \\
\hline Hawaii & $224(60.2)$ & $148(39.8)$ & $1.81(1.43-2.28)$ \\
\hline lowa & $651(69.5)$ & $286(30.5)$ & $1.15(0.98-1.35)$ \\
\hline Kentucky & $1,066(73.0)$ & $394(27.0)$ & $0.90(0.79-1.03)$ \\
\hline Louisiana & $1,278(80.0)$ & $320(20.0)$ & $0.59(0.51-0.67)$ \\
\hline New Jersey & $1,994(75.8)$ & $637(24.2)$ & $0.84(0.76-0.94)$ \\
\hline New Mexico & $292(56.8)$ & $222(43.2)$ & $2.17(1.79-2.63)$ \\
\hline Seattle-Puget Sound & $627(54.3)$ & $527(45.7)$ & $2.13(1.87-2.43)$ \\
\hline Utah & $309(71.2)$ & $125(28.8)$ & $0.91(0.73-1.14)$ \\
\hline \multicolumn{4}{|l|}{ Race } \\
\hline White & $11,980(69.8)$ & $5,193(30.2)$ & 1.00 [Ref] \\
\hline Black & $2,422(71.4)$ & $969(28.6)$ & $0.97(0.88-1.06)$ \\
\hline Other & $1,297(67.8)$ & $617(32.2)$ & $0.95(0.85-1.06)$ \\
\hline Unknown & 49 (76.6) & $15(23.4)$ & $0.59(0.32-1.07)$ \\
\hline \multicolumn{4}{|l|}{ Diagnosis year } \\
\hline 2010 & $4,246(75.4)$ & $1,386(24.6)$ & 1.00 [Ref] \\
\hline 2011 & $3,998(70.5)$ & $1,670(29.5)$ & $1.31(1.20-1.42)$ \\
\hline 2012 & $3,854(68.2)$ & $1,798(31.8)$ & $1.46(1.34-1.59)$ \\
\hline 2013 & $3,650(65.3)$ & $1,940(34.7)$ & $1.65(1.52-1.80)$ \\
\hline \multicolumn{4}{|l|}{ Insurance } \\
\hline Private/Medicare & $12,022(69.4)$ & $5,301(30.6)$ & 1.00 [Ref] \\
\hline Medicaid & $2,521(71.4)$ & 1,009 (28.6) & $0.82(0.76-0.90)$ \\
\hline Uninsured & $860(69.9)$ & $370(30.1)$ & $0.79(0.69-0.90)$ \\
\hline Unknown & $345(75.2)$ & $114(24.8)$ & $0.88(0.70-1.11)$ \\
\hline \multicolumn{4}{|l|}{ Marital status } \\
\hline Married & $7,491(66.6)$ & $3,759(33.4)$ & 1.00 [Ref] \\
\hline $\begin{array}{l}\text { Divorced/ } \\
\text { Separated }\end{array}$ & $1,853(69.2)$ & $826(30.8)$ & $0.87(0.79-0.96)$ \\
\hline Widowed & $2,496(79.2)$ & $654(20.8)$ & $0.85(0.76-0.94)$ \\
\hline Single/Never married & $3,000(69.8)$ & $1,300(30.2)$ & $0.79(0.73-0.86)$ \\
\hline Unknown & $908(78.1)$ & $255(21.9)$ & $0.59(0.50-0.68)$ \\
\hline
\end{tabular}

\begin{tabular}{|c|c|c|c|}
\hline & $\begin{array}{c}\text { No KRAS Testing } \\
(\mathrm{N}=15,748) \\
\mathrm{N}(\%)\end{array}$ & $\begin{array}{c}\text { KRAS Testing } \\
(\mathrm{N}=6,794) \\
\mathrm{N}(\%)\end{array}$ & $\begin{array}{c}\text { aOR }^{a} \\
(95 \% \mathrm{Cl})\end{array}$ \\
\hline \multicolumn{4}{|l|}{ Residence (1 missing) } \\
\hline Metropolitan area & $13,652(69.5)$ & $5,983(30.5)$ & $1.00[$ Ref] \\
\hline $\begin{array}{l}\text { Nonmetropolitan } \\
\text { area/rural }\end{array}$ & $2,096(72.1)$ & $810(27.9)$ & $0.85(0.77-0.94)$ \\
\hline \multicolumn{4}{|l|}{ Subsite } \\
\hline Right-side colon & $6,433(70.4)$ & $2,701(29.6)$ & $1.00(0.94-1.07)$ \\
\hline Left-side colon & $6,077(68.1)$ & 2,849 (31.9) & $1.00[$ Ref] \\
\hline Rectum & $3,238(72.2)$ & $1,244(27.8)$ & $0.86(0.79-0.94)$ \\
\hline \multicolumn{4}{|l|}{ Histology } \\
\hline Adenocarcinoma & $14,254(69.9)$ & $6,150(30.1)$ & $1.00[$ Ref] \\
\hline $\begin{array}{l}\text { Mucinous } \\
\text { adenocarcinoma }\end{array}$ & $1,494(69.9)$ & $644(30.1)$ & $0.98(0.88-1.09)$ \\
\hline \multicolumn{4}{|l|}{ Grade } \\
\hline Well differentiated (I) & $650(70.1)$ & $277(29.9)$ & $1.00[$ Ref] \\
\hline $\begin{array}{l}\text { Moderately } \\
\text { differentiated (II) }\end{array}$ & $8,484(69.0)$ & $3,817(31.0)$ & $0.99(0.85-1.15)$ \\
\hline $\begin{array}{l}\text { Poorly differentiated } \\
\text { (III) }\end{array}$ & $3,099(68.4)$ & $1,431(31.6)$ & $0.99(0.84-1.17)$ \\
\hline Undifferentiated (IV) & $481(65.6)$ & $252(34.4)$ & $1.06(0.85-1.32)$ \\
\hline Unknown & $3,034(74.9)$ & $1,017(25.1)$ & $0.83(0.70-0.98)$ \\
\hline \multicolumn{4}{|l|}{ T stage } \\
\hline$\leq \mathrm{T} 3$ & $7,556(69.3)$ & $3,347(30.7)$ & $1.00[$ Ref] \\
\hline$\geq \mathrm{T} 4 \mathrm{a}$ & $4,364(67.3)$ & $2,124(32.7)$ & $1.04(0.97-1.12)$ \\
\hline TX (unknown) & $3,828(74.3)$ & $1,323(25.7)$ & $0.95(0.87-1.05)$ \\
\hline \multicolumn{4}{|l|}{$\mathrm{N}$ stage } \\
\hline NO & $5,016(73.2)$ & $1,840(26.8)$ & 1.00 [Ref] \\
\hline$\geq \mathrm{N} 1 \mathrm{a}$ & $8,759(66.5)$ & $4,404(33.5)$ & $1.16(1.07-1.24)$ \\
\hline NX (Unknown) & $1,973(78.2)$ & $550(21.8)$ & $0.77(0.69-0.87)$ \\
\hline \multicolumn{4}{|l|}{ M stage/metastatic sites } \\
\hline $\begin{array}{l}\text { M1a: liver or lung } \\
\text { only }\end{array}$ & $6,768(69.3)$ & $2,998(30.7)$ & $1.00[$ Ref $]$ \\
\hline $\begin{array}{l}\text { M1a: bone or brain } \\
\text { only }\end{array}$ & $128(79.5)$ & $33(20.5)$ & $0.68(0.45-1.01)$ \\
\hline $\begin{array}{l}\text { M1a: other single } \\
\text { organ/site }\end{array}$ & $1,125(75.1)$ & $374(25.0)$ & $0.74(0.65-0.85)$ \\
\hline $\begin{array}{l}\text { M1b: multiple } \\
\text { organs/sites }\end{array}$ & $6,780(68.4)$ & $3,139(31.7)$ & $1.05(0.98-1.12)$ \\
\hline M1 NOS & $947(79.1)$ & $250(20.9)$ & $0.67(0.57-0.78)$ \\
\hline \multicolumn{4}{|c|}{ Was stage IV CRC the only cancer ever diagnosed? } \\
\hline Yes & $12,318(68.7)$ & $5,601(31.3)$ & $1.08(1.00-1.17)$ \\
\hline No & $3,430(74.2)$ & $1,193(25.8)$ & 1.00 [Ref] \\
\hline \multicolumn{4}{|l|}{ Surgery } \\
\hline Yes & $8,733(74.2)$ & $4,221(25.8)$ & $1.05(0.96-1.14)$ \\
\hline No or unknown & $7,015(67.4)$ & $2,573(32.6)$ & $1.00[$ Ref] \\
\hline \multicolumn{4}{|l|}{ Radiation } \\
\hline Yes or recommended & $2,019(73.2)$ & $814(26.8)$ & $0.90(0.82-1.00)$ \\
\hline No/Unknown/Refused & $13,729(71.3)$ & $5,980(28.7)$ & 1.00 [Ref] \\
\hline
\end{tabular}

Bolded values represent adjusted odds ratios that were statistically significant (ie, $95 \% \mathrm{Cl}$ did not contain 1.00 ).

Abbreviations: aOR, adjusted odds ratio; CRC, colorectal cancer; NOS, not otherwise specified.

adds ratios are adjusted for all variables in table.

\section{Sidedness}

Rectal cancers were excluded from sidedness analyses, leaving 18,060 patients with colon cancer: $49 \%$ with left-sided and $51 \%$ with right-sided. Of those KRAS-tested, $37 \%$ of patients with left-sided cancer were KRAS-mutant compared with $52 \%$ of those with right-sided. Table 3 displays data in a manner analogous to Table 2 by right-versus left-sided, in which the dependent variable was right-sided cancer and the independent variables included patient and tumor characteristics in Table 3. The proportions of those with right-sided cancer most substantially varied by age ( $31 \%$ of those $<30$ years vs $64 \%$ of $\geq 80$ years), histology (64\% mucinous vs $49 \%$ nonmucinous), grade ( $47 \%$ well-differentiated vs $63 \%$ undifferentiated), and radiation ( $32 \%$ yes vs $52 \%$ no). In the logistic model, variables associated with rightsided cancer were older age, female sex, black race, mucinous histology, poorly or undifferentiated grade, $\mathrm{N}$ stage $\geq \mathrm{N} 1 \mathrm{a}$, metastasis to other single site (not 


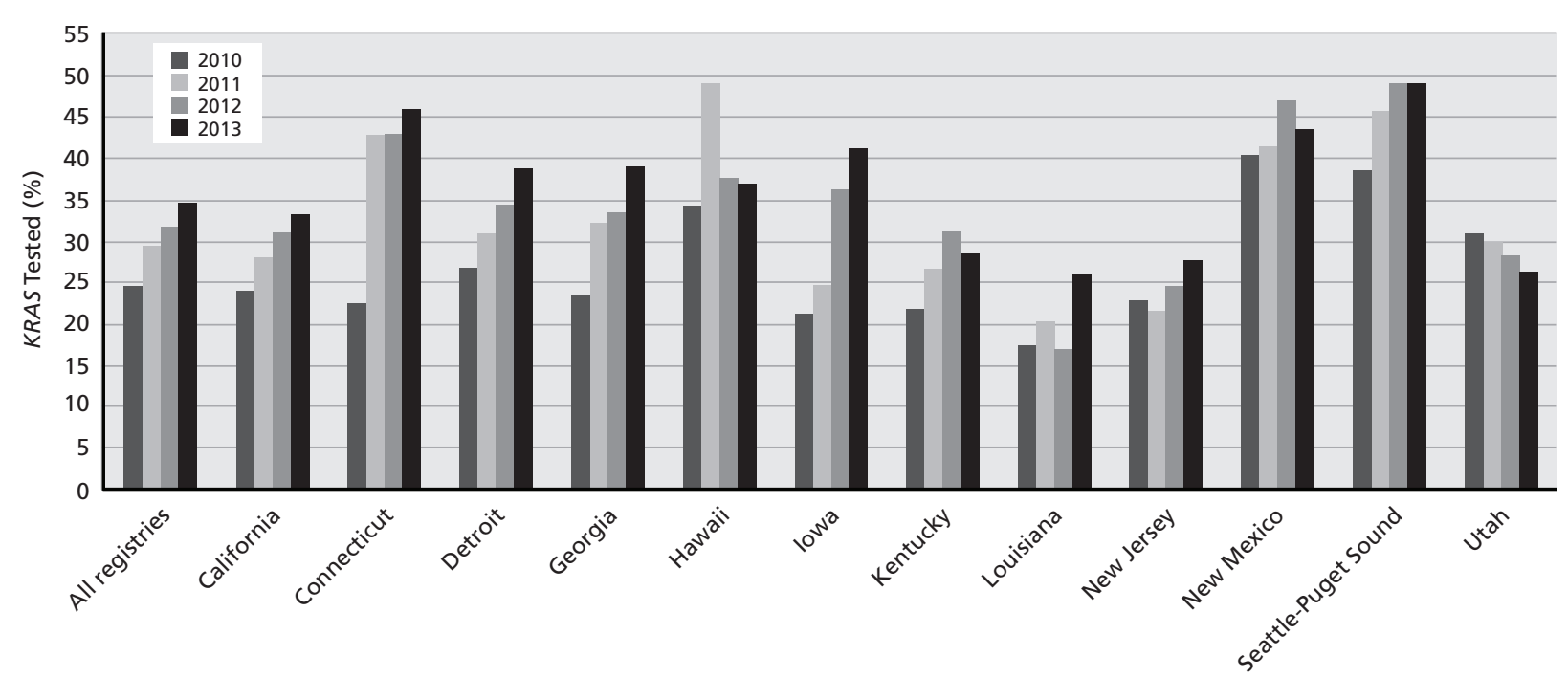

Figure 1. KRAS testing rates by SEER registry and year.

liver, lung, bone, or brain) or to multiple organs, and history of multiple cancers (Table 3 ).

\section{Survival}

A total of 16,952 patients with CRC were diagnosed in 2010-2012 and were included in survival analyses. Of those, $46 \%(n=7,743)$ died within 12 months of diagnosis, $50 \%(n=8,438)$ survived $>12$ months, and $5 \%(n=771)$ had $<12$ months of follow-up but were last known to be alive. KRAS testing rates across these groups differed significantly: 21\% versus 35\% versus $33 \%$, respectively $(P<.0001)$. The proportion of right-sided cancer across groups also differed significantly: $57 \%$ versus $44 \%$ versus $51 \%$, respectively $(P<.0001)$.

Among all 16,952 patients with CRC, Cox models showed the following variables were associated with increased hazard of death: older age; male sex; black race; earlier diagnosis year; Medicaid or no insurance; single, divorced, or widowed; poorly or undifferentiated grade; $\mathrm{T}$ stage $\geq \mathrm{T} 4 \mathrm{a}$ or unknown; $\mathrm{N}$ stage $\geq \mathrm{N} 1 \mathrm{a}$ or unknown; metastasis to bone or brain only, multiple organs, or NOS; no surgery; no radiation; and no KRAS testing (Table 4). Compared with left-sided cancer, those with right-sided cancer had a greater risk of death (HR, 1.27; 95\% CI, 1.22-1.32) and those with rectal cancer had a lower risk (HR, 0.90; 95\% CI, 0.85-0.96). No significant difference in risk was seen between those with KRAS-mutant versus KRAS-WT cancer.
Cox models were run on the subset of patients who had KRAS testing $(n=4,854)$, and similar relationships emerged as in the model with the 16,952 patients. KRAS mutations were not associated with risk of death, whereas right-sided cancer was associated with a greater risk of death compared with leftsided and rectal cancer (Table 4). However, when separate models were run for the 2,064 patients with left-sided and the 1,930 patients with rightsided cancer, having a KRAS mutation was associated with greater risk of death among those with left-sided cancer (HR, 1.18; 95\% CI, 1.05-1.33) but not right-sided (HR, 0.93; 95\% CI, 0.83-1.03). In both left- and right-sided models, advanced age ( $\geq 80$ years), single/never married, higher grade, $T$ stage $\geq 4 \mathrm{a}$, $M$ stage $1 \mathrm{~b}$, and no surgical treatment were associated with increased hazard of death ( $\mathrm{Ta}$ ble 5). For right-sided only, those diagnosed in earlier years had a greater risk of death; for left-sided only, black race, Medicaid or no insurance, widowed, unknown $\mathrm{N}$ stage, and metastases NOS were associated with a greater risk of death.

\section{Discussion}

Despite NCCN recommending all patients with stage IV CRC be tested for KRAS mutations at time of diagnosis, only $30 \%$ of patients diagnosed in 2010-2013 were tested according to SEER data. Overall testing rates increased from 25\% in 2010 to $35 \%$ in 2013 , and there was variation by registry, 
KRAS Status and Tumor Location in Stage IV CRC

Table 3. Patient, Tumor, and Treatment Characteristics and Odds of Having Right-Sided Versus Left-Sided Colon Cancer $\left(\mathrm{N}=18,060^{\circ}\right)$

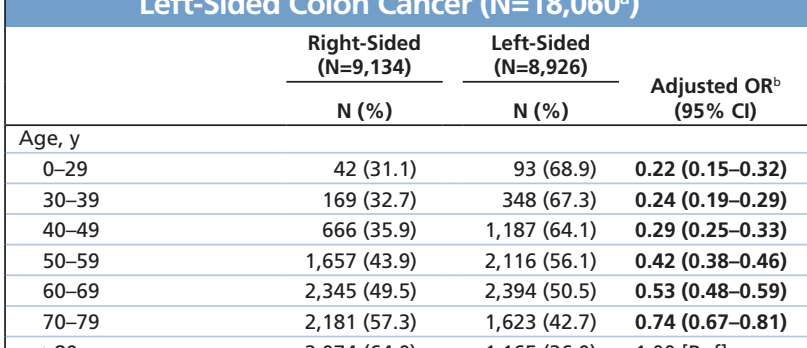

$\begin{array}{llll}\geq 80 & 2,074(64.0) & 1,165(36.0) & 1.00 \text { [Ref] }\end{array}$

Sex

\begin{tabular}{|llll|}
\hline Female & $4,679(55.2)$ & $3,798(44.8)$ & $\mathbf{1 . 3 5 ( 1 . 2 7 - 1 . 4 4 )}$ \\
\hline Male & $4,455(46.5)$ & $5,128(53.5)$ & $1.00[$ Ref] \\
\hline Race & & & \\
\hline
\end{tabular}

Race

\begin{tabular}{lrrl} 
White & $6,938(50.9)$ & $6,682(49.1)$ & $1.00[$ Ref] \\
\hline Black & $1,611(55.8)$ & $1,275(44.2)$ & $\mathbf{1 . 4 5 ( 1 . 3 3 - 1 . 5 8 )}$ \\
\hline Other & $565(37.6)$ & $936(62.4)$ & $\mathbf{0 . 6 2 ( 0 . 5 5 - 0 . 6 9 )}$
\end{tabular}

\begin{tabular}{|c|c|}
\hline & $20(37$ \\
\hline
\end{tabular}

Diagnosis year

$2010 \quad 2,341(51.1) \quad 2,243(48.9) \quad 1.00$ [Ref]

$20112,283(50.3) \quad 2,256(49.7) \quad 0.99(0.90-1.07)$

$2012 \quad 2,290(50.2) \quad 2,268(49.8) \quad 1.00(0.92-1.09)$

$2013 \quad 2,220(50.7) \quad 2,159(49.3) \quad 1.04(0.96-1.14)$

Histology

Adenocarcinoma $\quad 7,942(49.0) \quad 8,256(51.0) \quad 1.00$ [Ref]

$\begin{array}{llll}\begin{array}{l}\text { Mucinous } \\ \text { adenocarcinoma }\end{array} & 1,192(64.0) & 670(36.0) & \mathbf{1 . 6 6 ( 1 . 4 9 - 1 . 8 4 )}\end{array}$

Grade

Well differentiated (I) $344(46.9) \quad 390(53.1) \quad 1.00[$ Ref]

Moderately $\quad 4,590(47.2) \quad 5,137(52.8) \quad 1.06(0.90-1.24)$

differentiated (II)

Poorly differentiated (III) $\quad 2,365(61.6) \quad 1,473(38.4) \quad \mathbf{1 . 7 5}(\mathbf{1} .48-\mathbf{2 . 0 7})$

$\begin{array}{llll}\text { Undifferentiated (IV) } & 427(63.2) & 249(36.8) & \mathbf{1 . 7 4}(\mathbf{1 . 3 9}-\mathbf{2 . 1 7})\end{array}$

$\begin{array}{llll}\text { Unknown } & 1,408(45.6) & 1,677(54.4) & 1.05(0.89-1.25)\end{array}$

T stage

$\begin{array}{llll}\leq \mathrm{T} 3 & 4,202(50.0) & 4,211(50.1) & 1.00[\mathrm{Ref}]\end{array}$

$\geq \mathrm{T} 4 \mathrm{a} \quad 3,142(54.4) \quad 2,631(45.6) \quad 1.03(0.95-1.10)$

$\begin{array}{llll}\text { TX (unknown) } & 1,790(46.2) & 2,084(53.8) & 1.00(0.91-1.10)\end{array}$

$\mathrm{N}$ stage

$\begin{array}{llll}\text { N0 } & 2,281(43.7) & 2,940(56.3) & 1.00[\text { Ref] } \\ \geq \text { N1a } & 5,986(54.7) & 4,956(45.3) & \mathbf{1 . 5 5 ( 1 . 4 4 - 1 . 6 7 )}\end{array}$

NX (Unknown) $\quad 867(45.7) \quad 1,030(54.3) \quad 1.10(0.98-1.23)$

$\mathrm{M}$ stage/metastatic sites

M1a: liver or lung only $\quad 3,755(48.1) \quad 4,057(51.9) \quad 1.00$ [Ref]

M1a: bone or brain only $\quad 47(48.5) \quad 50(51.6) \quad 0.94(0.62-1.43)$

M1a: other single $\quad 673(55.7) \quad 536(44.3) \quad 1.15(1.01-1.31)$

M1b: multiple organs/ $\quad 4,262(52.8) \quad 3,816(47.2) \quad 1.13(1.06-1.21)$

M1b: multiple organs/ $\quad 4,262(52.8) \quad 3,816(47.2) \quad 1.13(1.06-1.21)$
sites

$\begin{array}{llll}\text { M1 NOS } & 397(46.0) & 467(54.1) & \mathbf{0 . 8 6}(\mathbf{0 . 7 4 - 0 . 9 9 )}\end{array}$

Was stage IV CRC the only cancer ever diagnosed?

\begin{tabular}{|c|c|c|c|}
\hline Yes & $7,015(49.3)$ & $7,225(50.7)$ & $0.91(0.85-0.99)$ \\
\hline No & $2,119(55.5)$ & $1,701(44.5)$ & 1.00 [Ref] \\
\hline \multicolumn{4}{|l|}{ Surgery } \\
\hline Yes & $6,121(53.4)$ & $5,335(46.6)$ & \multirow{2}{*}{$\begin{array}{l}\text { Not included } \\
\text { in model }\end{array}$} \\
\hline No or unknown & $3,013(45.6)$ & $3,591(54.4)$ & \\
\hline \multicolumn{4}{|l|}{ Radiation } \\
\hline Yes or recommended & $314(31.5)$ & $683(68.5)$ & \multirow{2}{*}{$\begin{array}{l}\text { Not included } \\
\text { in model }\end{array}$} \\
\hline No/Unknown/Refused & $8,820(51.7)$ & $8,243(48.3)$ & \\
\hline \multicolumn{4}{|l|}{ KRAS test } \\
\hline Normal & $1,300(42.1)$ & $1,790(57.9)$ & \multirow{3}{*}{$\begin{array}{l}\text { Not included } \\
\text { in model }\end{array}$} \\
\hline Mutated & $1,401(57.0)$ & $1,059(43.1)$ & \\
\hline No testing done & $6,433(51.4)$ & $6,077(48.6)$ & \\
\hline
\end{tabular}

Bolded values represent adjusted odds ratios that were statistically significant (ie, $95 \% \mathrm{Cl}$ did not contain 1.00).

Abbreviations: CRC, colorectal cancer; NOS, not otherwise specified.

${ }^{a}$ Excludes rectal cancer.

bOdds ratios adjusted for all variables in table unless otherwise indicated. with almost half of the patients diagnosed in 2013 having documentation of testing in Seattle-Puget Sound compared with only one-quarter of patients in Louisiana. Similar to our findings from 2010, patients recorded as having been testing tended to be younger, married, and living in metropolitan areas. ${ }^{11}$ However, the current analysis also showed that those with private insurance or Medicare were more likely to receive testing than those with Medicaid or no insurance. Given the high cost of anti-EGFR therapy-approximately $\$ 25,000$ USD for an 8-week cycle-there may be disparities in who is offered KRAS testing based on ability to pay for anti-EGFR therapy. ${ }^{28}$ Patients recorded as not having KRAS testing had a greater risk of death after controlling for other factors. This could potentially reflect that those tested received higher-quality care and/or were offered EGFR inhibitors, which extended their lives. It is also possible oncologists were less likely to recommend KRAS testing for patients with a poor prognosis, or patients with a poor prognosis may have declined to receive targeted therapy and therefore were not tested.

Among those who received KRAS testing according to SEER data, KRAS mutations occurred more frequently in patients with right-sided cancer, which is consistent with previous findings. ${ }^{22,29} \mathrm{Sim}$ ilar to an evaluation of European clinical trial patients by Missiaglia et $\mathrm{al},{ }^{18}$ we found that right-sided cancer was significantly associated with poorly differentiated tumors of mucinous histology penetrating to the surface of the visceral peritoneum or beyond $(\geq \mathrm{T} 4 \mathrm{a})$, lymph node metastasis $(\geq \mathrm{N} 1 \mathrm{a})$, and metastasis to $>1$ organ/site or the peritoneum (stage IVB). In addition, among those diagnosed with stage IV colon cancer, blacks were more likely to have rightsided cancer. Other studies examining all stages of colon cancer have detected an association between black race and right-sided cancer. ${ }^{30,31}$

In Cox models including patients with left- or right-sided cancers, right-sided cancer was associated with greater risk of death after controlling for patient/tumor characteristics, which is consistent with findings from a recent meta-analysis. ${ }^{32}$ One possible explanation is that right-sided cancer is associated with increased BRAF mutations, which are associated with worse prognosis and poorer response to cetuximab. ${ }^{33}$ BRAF mutation status was not available in SEER data, so we were unable to explore this as 
Charlton et al

\begin{tabular}{|c|c|c|}
\hline \multicolumn{3}{|c|}{$\begin{array}{r}\text { Table 4. HRs for All Pa } \\
\text { KRAS Testing }\end{array}$} \\
\hline & \multicolumn{2}{|c|}{$\begin{array}{c}\text { Adjusted HR } \mathrm{HR}^{\mathrm{a}} \\
(95 \% \mathrm{Cl})\end{array}$} \\
\hline & $\begin{array}{l}\text { All Patients } \\
(\mathrm{N}=16,952)\end{array}$ & $\begin{array}{l}\text { KRAS-Tested } \\
\text { Patients Only } \\
(\mathrm{N}=4,854)\end{array}$ \\
\hline \multicolumn{3}{|l|}{ KRAS test } \\
\hline Normal & $1.00[$ Ref $]$ & $1.00[$ Ref] \\
\hline Mutated & $1.04(0.97-1.12)$ & $1.04(0.96-1.12)$ \\
\hline No testing done & $1.31(1.25-1.39)$ & - \\
\hline \multicolumn{3}{|l|}{ Age, y } \\
\hline $0-29$ & 1.00 [Ref] & 1.00 [Ref] \\
\hline $30-39$ & $0.98(0.77-1.26)$ & $1.13(0.77-1.67)$ \\
\hline $40-49$ & $1.01(0.80-1.26)$ & $1.10(0.77-1.58)$ \\
\hline $50-59$ & $1.16(0.93-1.45)$ & $1.28(0.90-1.83)$ \\
\hline $60-69$ & $1.39(1.11-1.73)$ & $1.51(1.06-2.15)$ \\
\hline $70-79$ & $1.83(1.46-2.28)$ & $1.80(1.25-2.57)$ \\
\hline$\geq 80$ & $2.83(2.26-3.54)$ & $2.73(1.89-3.96)$ \\
\hline \multicolumn{3}{|l|}{ Race } \\
\hline White & 1.00 [Ref] & 1.00 [Ref] \\
\hline Black & $1.11(1.06-1.17)$ & $1.18(1.06-1.30)$ \\
\hline Other & $0.92(0.86-0.99)$ & $0.94(0.85-1.03)$ \\
\hline \multicolumn{3}{|l|}{ Diagnosis year } \\
\hline 2010 & 1.00 [Ref] & 1.00 [Ref] \\
\hline 2011 & $0.99(0.95-1.03)$ & $0.95(0.87-1.04)$ \\
\hline 2012 & $0.94(0.89-0.98)$ & $0.93(0.84-1.03)$ \\
\hline \multicolumn{3}{|l|}{ Insurance } \\
\hline Private/Medicare & 1.00 [Ref] & 1.00 [Ref] \\
\hline Medicaid & $1.24(1.18-1.31)$ & $1.19(1.07-1.32)$ \\
\hline Uninsured & $1.32(1.21-1.43)$ & $1.25(1.06-1.47)$ \\
\hline Unknown & $1.10(0.97-1.25)$ & $1.23(0.92-1.64)$ \\
\hline \multicolumn{3}{|l|}{ Marital status } \\
\hline Married & $1.00[\operatorname{Ref}]$ & $1.00[\operatorname{Ref}]$ \\
\hline Divorced/Separated & $1.10(1.04-1.17)$ & $1.11(0.98-1.23)$ \\
\hline Widowed & $1.19(1.12-1.26)$ & $1.27(1.12-1.43)$ \\
\hline Single/Never married & $1.20(1.14-1.27)$ & $1.23(1.12-1.36)$ \\
\hline Unknown & $1.00(0.92-1.09)$ & $0.85(0.69-1.05)$ \\
\hline
\end{tabular}

\begin{tabular}{|c|c|c|}
\hline & \multicolumn{2}{|c|}{$\begin{array}{l}\text { Adjusted } \mathrm{HR}^{\mathrm{a}} \\
(95 \% \mathrm{Cl})\end{array}$} \\
\hline & $\begin{array}{l}\text { All Patients } \\
(\mathrm{N}=16,952)\end{array}$ & $\begin{array}{l}\text { KRAS-Tested } \\
\text { Patients Only } \\
(\mathrm{N}=4,854)\end{array}$ \\
\hline \multicolumn{3}{|l|}{ Subsite } \\
\hline Right side colon & $1.27(1.22-1.32)$ & $1.39(1.28-1.51)$ \\
\hline Left side colon & $1.00[$ Ref] & $1.00[$ Ref] \\
\hline Rectum & $0.90(0.85-0.96)$ & $0.90(0.81-1.01)$ \\
\hline \multicolumn{3}{|l|}{ Grade } \\
\hline Well differentiated (I) & $1.00[$ Ref] & 1.00 [Ref] \\
\hline Moderately differentiated (II) & $0.97(0.89-1.07)$ & $1.05(0.87-1.27)$ \\
\hline Poorly differentiated (III) & $1.43(1.30-1.58)$ & $1.68(1.38-2.05)$ \\
\hline Undifferentiated (IV) & $1.56(1.37-1.78)$ & $1.85(1.44-2.39)$ \\
\hline Unknown & $1.13(1.02-1.24)$ & $1.25(1.02-1.53)$ \\
\hline \multicolumn{3}{|l|}{ T stage } \\
\hline$\leq \mathrm{T} 3$ & 1.00 [Ref] & 1.00 [Ref] \\
\hline$\geq T 4 a$ & $1.26(1.20-1.32)$ & $1.23(1.13-1.34)$ \\
\hline TX (unknown) & $1.14(1.07-1.20)$ & $1.09(0.97-1.22)$ \\
\hline \multicolumn{3}{|l|}{$\mathrm{N}$ stage } \\
\hline No & $1.00[$ Ref] & 1.00 [Ref] \\
\hline$\geq \mathrm{N} 1 \mathrm{a}$ & $1.10(1.05-1.15)$ & $1.07(0.97-1.17)$ \\
\hline NX (unknown) & $1.17(1.10-1.25)$ & $1.15(1.00-1.32)$ \\
\hline \multicolumn{3}{|l|}{ M stage/metastatic sites } \\
\hline M1a: liver or lung only & $1.00[$ Ref $]$ & - \\
\hline M1a: bone or brain only & $1.77(1.45-2.18)$ & - \\
\hline M1a: other single organ/site & $0.78(0.72-0.85)$ & $1.00[\text { Ref }]^{\mathrm{b}}$ \\
\hline M1b: multiple organs/sites & $1.37(1.32-1.43)$ & $1.42(1.31-1.53)$ \\
\hline M1 NOS & $1.28(1.18-1.39)$ & $1.21(0.98-1.49)$ \\
\hline \multicolumn{3}{|l|}{ Surgery } \\
\hline Yes & $0.48(0.46-0.51)$ & $0.52(0.47-0.58)$ \\
\hline No or Unknown & 1.00 [Ref] & $1.00[$ Ref] \\
\hline \multicolumn{3}{|l|}{ Radiation } \\
\hline Yes or recommended & $0.75(0.70-0.80)$ & NS \\
\hline No/Unknown/Refused & 1.00 [Ref] & NS \\
\hline \multicolumn{3}{|l|}{ Sex } \\
\hline Female & $0.91(0.87-0.94)$ & NS \\
\hline Male & 1.00 [Ref] & NS \\
\hline
\end{tabular}

Bolded values represent adjusted odds ratios that were statistically significant (ie, $95 \% \mathrm{Cl}$ did not contain 1.00 ).

Abbreviations: CRC, colorectal cancer; HR, hazard ratio; NOS, not otherwise specified; NS, nonsignificant.

aHRs were determined using manual backward selection to retain only those variables significant at $P<.05$, except the following variables which were forced into each model: KRAS results, age, race, diagnosis year, insurance, marital status, $\mathrm{T}$ stage, $\mathrm{N}$ stage, and $\mathrm{M}$ stage/metastatic sites.

${ }^{b}$ Due to small numbers, metastatic sites were not included in the KRAS-tested patients-only model; overall M stage was used.

a potential driver of poorer survival among patients with right-sided cancer.

In the overall survival analysis of patients who received KRAS testing, we did not find a survival advantage for those with KRAS-WT tumors compared with KRAS-mutant tumors. We did, however, find a survival advantage for KRAS-WT tumors within the population of patients with left-sided tumors. One explanation for not finding a survival advantage in the overall study population (not stratified by tumor location) may be that EGFR inhibitors are only effective in left-sided tumors and not right-sided tumors. This explanation is supported by a retrospective analysis by Venook et $\mathrm{al}^{34}$ of the ongoing phase III CALGB/SWOG 80405 trial (ClinicalTrials.gov identifier: NCT00265850), ${ }^{35}$ which found that among patients who received cetuximab, those with left-sided tumors survived 37.5 months compared with 16.4 months among those with right-sided tumors. Furthermore, they found among those with right-sided tumors, treatment with bevacizumab was associated with longer survival compared with cetuximab (24.5 vs 16.4 months); conversely, among patients with left-sided tumors, treatment with cetuximab was associated with longer survival compared with bevacizumab (37.5 vs 32.1 months). Our results are consistent with the Venook study findings and support the concept that patients with left-sided KRAS-WT primary tumors are more likely to benefit from anti-EGFR targeted therapy, and therefore will have better outcomes than those with left-sided KRAS-mutant primary 
KRAS Status and Tumor Location in Stage IV CRC

Table 5. Hazard Ratios for Patients Who Received KRAS Testing: Right- Versus Left-Sided

\begin{tabular}{|c|c|c|}
\hline & $\begin{array}{l}\text { Right-Sided } \\
(\mathrm{N}=1,930)\end{array}$ & $\begin{array}{l}\text { Left-Sided } \\
(\mathrm{N}=2,064)\end{array}$ \\
\hline & $\begin{array}{l}\text { Adjusted HR } \\
(95 \% \mathrm{Cl})\end{array}$ & $\begin{array}{c}\text { Adjusted HR } \\
(95 \% \mathrm{Cl})\end{array}$ \\
\hline \multicolumn{3}{|l|}{ KRAS test } \\
\hline Normal & 1.00 [Ref] & $1.00[$ Ref] \\
\hline Mutated & $0.93(0.83-1.03)$ & $1.18(1.05-1.33)$ \\
\hline \multicolumn{3}{|l|}{ Age, y } \\
\hline $0-29$ & 1.00 [Ref] & $1.00[$ Ref] \\
\hline $30-39$ & $0.88(0.41-1.89)$ & $1.09(0.64-1.87)$ \\
\hline $40-49$ & $0.91(0.44-1.87)$ & $1.14(0.69-1.90)$ \\
\hline $50-59$ & $1.06(0.52-2.15)$ & $1.40(0.85-2.30)$ \\
\hline $60-69$ & $1.29(0.63-2.62)$ & $1.51(0.92-2.49)$ \\
\hline $70-79$ & $1.44(0.71-2.93)$ & $1.93(1.16-3.22)$ \\
\hline$\geq 80$ & $2.17(1.05-4.45)$ & $2.94(1.72-5.01)$ \\
\hline \multicolumn{3}{|l|}{ Race } \\
\hline White & 1.00 [Ref] & 1.00 [Ref] \\
\hline Black & $0.98(0.85-1.15)$ & $1.39(1.18-1.63)$ \\
\hline Other & $0.81(0.64-1.03)$ & $0.88(0.73-1.06)$ \\
\hline \multicolumn{3}{|l|}{ Diagnosis year } \\
\hline 2010 & 1.00 [Ref] & 1.00 [Ref] \\
\hline 2011 & $0.89(0.79-1.02)$ & $1.03(0.89-1.18)$ \\
\hline 2012 & $0.84(0.73-0.97)$ & $1.01(0.86-1.18)$ \\
\hline \multicolumn{3}{|l|}{ Insurance } \\
\hline Private/Medicare & $1.00[$ Ref $]$ & $1.00[$ Ref $]$ \\
\hline Medicaid & $1.18(0.99-1.40)$ & $1.26(1.07-1.47)$ \\
\hline Uninsured & $1.32(0.99-1.75)$ & $1.34(1.04-1.73)$ \\
\hline Unknown & $1.11(0.66-1.87)$ & $1.21(0.79-1.86)$ \\
\hline \multicolumn{3}{|l|}{ Marital status } \\
\hline Married & $1.00[$ Ref] & $1.00[$ Ref] \\
\hline Divorced/separated & $1.05(0.88-1.25)$ & $1.05(0.87-1.26)$ \\
\hline Widowed & $1.18(1.00-1.39)$ & $1.24(1.00-1.54)$ \\
\hline Single/never married & $1.23(1.05-1.45)$ & $1.24(1.06-1.44)$ \\
\hline Unknown & $0.93(0.68-1.29)$ & $0.87(0.62-1.22)$ \\
\hline \multicolumn{3}{|l|}{ Grade } \\
\hline Well differentiated (I) & 1.00 [Ref] & $1.00[$ Ref] \\
\hline Moderately differentiated (II) & $0.96(0.70-1.32)$ & $1.26(0.95-1.68)$ \\
\hline Poorly differentiated (III) & $1.40(1.02-1.93)$ & $2.05(1.51-2.78)$ \\
\hline Undifferentiated (IV) & $1.51(1.03-2.21)$ & $2.14(1.42-3.23)$ \\
\hline Unknown & $1.21(0.86-1.70)$ & $1.31(0.96-1.79)$ \\
\hline \multicolumn{3}{|l|}{ T stage } \\
\hline$\leq \mathrm{T} 3$ & $1.00[$ Ref $]$ & $1.00[$ Ref] \\
\hline$\geq \mathrm{T} 4 \mathrm{a}$ & $1.24(1.10-1.41)$ & $1.22(1.07-1.40)$ \\
\hline TX (Unknown) & $1.05(0.86-1.28)$ & $1.10(0.92-1.32)$ \\
\hline \multicolumn{3}{|l|}{$\mathrm{N}$ stage } \\
\hline NO & 1.00 [Ref] & $1.00[$ Ref] \\
\hline$\geq \mathrm{N} 1 \mathrm{a}$ & $1.14(0.98-1.32)$ & $1.08(0.93-1.25)$ \\
\hline NX (Unknown) & $1.24(0.98-1.58)$ & $1.25(1.01-1.55)$ \\
\hline \multicolumn{3}{|l|}{ M stage ${ }^{c}$} \\
\hline M1a & 1.00 [Ref] & 1.00 [Ref] \\
\hline $\mathrm{M} 1 \mathrm{~b}$ & $1.28(1.14-1.44)$ & $1.53(1.35-1.74)$ \\
\hline M1 NOS & $0.99(0.69-1.42)$ & $1.66(1.21-2.29)$ \\
\hline \multicolumn{3}{|l|}{ Surgery } \\
\hline Yes & $0.53(0.45-0.64)$ & $0.49(0.42-0.58)$ \\
\hline No or unknown & 1.00 [Ref] & 1.00 [Ref] \\
\hline
\end{tabular}

Bolded values represent adjusted odds ratios that were statistically significant (ie, $95 \% \mathrm{Cl}$ did not contain 1.00 ).

Abbreviations: CRC, colorectal cancer; HR, hazard ratio; NOS, not otherwise specified.

apatients with rectal cancer were excluded.

${ }^{b}$ Manual backward selection was used to retain variables significant at $P<.05$, except the following which were forced into all models: KRAS results, age, registry, race, diagnosis year, insurance, marital status,

T stage, N stage, and $\mathrm{M}$ stage.

'Due to small numbers, metastatic sites were not included in right- and left-sided models; overall M stage was used instead. tumors. In addition, our results suggest that patients with right-sided KRAS-WT primary tumors do not benefit from anti-EGFR targeted therapy, consistent with previous research, ${ }^{22,36}$ and therefore their outcomes will not be better than those with rightsided KRAS-mutant primary tumors. This may be due to more frequent active signaling via the EGFR pathway in left-sided compared with right-sided cancers, which may in turn make left-sided cancer more responsive to anti-EGFR therapy. ${ }^{18,37}$

Further, as previously mentioned, patients with right-sided tumors likely had more BRAF mutations, which are more resistant to anti-EGFR therapy. ${ }^{38-40}$ In contrast to anti-EGFR therapy, the effect of bevacizumab has been shown to be independent of tumor location. ${ }^{41}$ The nearly significant HR for KRAS-mutant right-sided tumors was 0.92 (95\% CI, 0.83-1.03) compared with KRAS-WT right-sided tumors, which could potentially suggest worse survival for those who received cetuximab instead of bevacizumab. This could explain why models containing patients with right- and left-sided tumors did not show a significant survival advantage associated with KRAS-WT status, and highlights the importance of stratification by tumor location.

Our study has several limitations. As previously mentioned, we did not have information about BRAF mutations. Also, the SEER program does not release data on chemotherapy in the public use data set due to concerns about the completeness of this information, and we cannot assume that all patients who received KRAS testing with a WT result received anti-EGFR therapy. As recommended by NCCN, KRAS testing may have occurred at time of diagnosis for planning purposes, ${ }^{42,43}$ but then traditional chemotherapy may have still been given as the first line of treatment due to physician or patient preference, or due to financial barriers associated with the very high cost of anti-EGFR therapy.

In addition, the KRAS variable is still relatively new, and increasing testing rates may be driven in part by more complete capture of KRAS information by registrars as they gain more experience in collecting this variable. Furthermore, a quality control study of the KRAS variable in Iowa and Louisiana patients found that coding guidelines were being applied somewhat inconsistently re- 
lated to timing of the testing. Some included instances of KRAS testing occurring several months postdiagnosis in determining second-course treatment, whereas others only counted testing within the first 4 months postdiagnosis per coding guidelines. ${ }^{42}$ Further, the primary source of information for cancer registries is hospital records. Given that KRAS mutation analysis is a referral test in most institutions and often ordered by oncologists, some test results may only be available in physician office records and not always available to registrars. ${ }^{6} \mathrm{Al}$ though these data issues may have some impact on the accuracy of the KRAS data, there is currently no other population-based source that can provide a more accurate assessment of KRAS testing across the nation.

Despite its limitations, this study has important strengths. We were able to assess KRAS testing rates across patient, tumor, and treatment characteristics, and examine relationships between KRAS status, sidedness, and survival using population-based data that avoided the selection bias inherent in the clinical trial setting. The SEER population includes an estimated $28 \%$ of cancers in the United States. ${ }^{44}$

\section{References}

1. American Cancer Society. Cancer Facts \& Figures 2016. Available at: https://www.cancer.org/research/cancer-facts-statistics/all-cancer-factsfigures/cancer-facts-figures-2016.html. Accessed November 6, 2017.

2. Howlader N, Noone AM, Krapcho M, et al. SEER Cancer Statistics Review 1975-2010, National Cancer Institute. Bethesda, MD. Available at: http://seer.cancer.gov/csr/1975_2010/. Accessed November 6, 2017.

3. Allegra CJ, Jessup JM, Somerfield MR, et al. American Society of Clinical Oncology provisional clinical opinion: testing for KRAS gene mutations in patients with metastatic colorectal carcinoma to predict response to anti-epidermal growth factor receptor monoclonal antibody therapy. J Clin Oncol 2009;27:2091-2096.

4. De Roock W, Jonker DJ, Di Nicolantonio F, et al. Association of KRAS p.G13D mutation with outcome in patients with chemotherapyrefractory metastatic colorectal cancer treated with cetuximab. JAMA 2010;304:1812-1820.

5. Lievre A, Bachet JB, Boige $\mathrm{V}$, et al. KRAS mutations as an independent prognostic factor in patients with advanced colorectal cancer treated with cetuximab. J Clin Oncol 2008;26:374-379.

6. Morton RF, Hammond EH. ASCO Provisional Clinical Opinion: KRAS, cetuximab, and panitumumab-clinical implications in colorectal cancer. J Oncol Pract 2009;5:71-72.

7. Vale CL, Tierney JF, Fisher D, et al. Does anti-EGFR therapy improve outcome in advanced colorectal cancer? A systematic review and metaanalysis. Cancer Treat Rev 2012;38:618-625.

8. Karapetis CS, Khambata-Ford S, Jonker DJ, et al. K-ras mutations and benefit from cetuximab in advanced colorectal cancer. N Engl J Med 2008;359:1757-1765.

9. Tejpar S, Peeters M, Humblet $\mathrm{Y}$, et al. Relationship of efficacy with KRAS status (wild type versus mutant) in patients with irinotecan-refractory metastatic colorectal cancer, treated with irinotecan and escalating doses of cetuximab: the EVEREST experience (preliminary data) [abstract]. J Clin Oncol 2008;26(Suppl 1):Abstract 4001.

\section{Conclusions}

Our results have several implications for future research. First, examining health disparities related to KRAS testing and subsequent treatment with antiEGFR therapy with respect to race, insurance status, and rurality is warranted. Similarly, exploring associations between right-sided cancer and black race among patients with stage IV cancer is critical given the poorer prognosis associated with right-sided cancer. Additionally, assessing other variables that could alter prognosis, such as MSI and BRAF, is important, but difficult to do on a population basis, because MSI and BRAF are not required elements for SEER registries. Through better understanding how sidedness and molecular characteristics of right- versus leftsided tumors affect response to therapy, patient response to treatment can be enhanced. Clinical trials in stage IV CRC should incorporate as much information as possible regarding tumor sidedness and various molecular tumor markers in order to determine optimal targeted therapy regimens.

10. Engstrom PF, Arnoletti JP, Benson AB III, et al. NCCN Clinical Practice Guidelines in Oncology: Colon Cancer. J Natl Compr Canc Netw 2009; 7:778-831.

11. Charlton ME, Karlitz JJ, Schlichting JA, et al. Factors associated with guideline-recommended KRAS testing in colorectal cancer patients: a population-based study. Am J Clin Oncol 2017;40:498-506.

12. Shen $H$, Yang J, Huang Q, et al. Different treatment strategies and molecular features between right-sided and left-sided colon cancers. World J Gastroenterol 2015;21:6470-6478.

13. Lee $\mathrm{GH}$, Malietzis $\mathrm{G}$, Askari A, et al. Is right-sided colon cancer different to left-sided colorectal cancer? A systematic review. Eur J Surg Oncol 2015;41:300-308

14. Powell AG, Wallace R, McKee RF, et al. The relationship between tumour site, clinicopathological characteristics and cancer-specific survival in patients undergoing surgery for colorectal cancer. Colorectal Dis 2012;14:1493-1499.

15. Azzoni C, Bottarelli L, Campanini N, et al. Distinct molecular patterns based on proximal and distal sporadic colorectal cancer: arguments for different mechanisms in the tumorigenesis. Int J Colorectal Dis 2007;22:115-126.

16. Glebov OK, Rodriguez LM, Nakahara K, et al. Distinguishing right from left colon by the pattern of gene expression. Cancer Epidemiol Biomarker Prev 2003;12:755-762.

17. Benedix F, Meyer F, Kube R, et al. Influence of anatomical subsite on the incidence of microsatellite instability, and KRAS and BRAF mutation rates in patients with colon carcinoma. Pathol Res Pract 2012;208:592597.

18. Missiaglia E, Jacobs B, D’Ario G, et al. Distal and proximal colon cancers differ in terms of molecular, pathological, and clinical features. Ann Oncol 2014;25:1995-2001.

19. Kalady MF, Dejulius KL, Sanchez JA, et al. BRAF mutations in colorectal cancer are associated with distinct clinical characteristics and worse prognosis. Dis Colon Rectum 2012;55:128-133. 
KRAS Status and Tumor Location in Stage IV CRC

20. Fric P, Sovova V, Sloncova E, Lojda Z, et al. Different expression of some molecular markers in sporadic cancer of the left and right colon. Eur J Cancer Prev 2000;9:265-268.

21. Elnatan J, Goh HS, Smith DR. C-KI-RAS activation and the biological behaviour of proximal and distal colonic adenocarcinomas. Eur J Cancer 1996;32a:491-497.

22. Brule SY, Jonker DJ, Karapetis CS, et al. Location of colon cancer (rightsided versus left-sided) as a prognostic factor and a predictor of benefit from cetuximab in NCIC CO.17. Eur J Cancer 2015;51:1405-1414.

23. Meguid RA, Slidell MB, Wolfgang CL, et al. Is there a difference in survival between right- versus left-sided colon cancers? Ann Surg Oncol 2008;15:2388-2394

24. Nawa T, Kato J, Kawamoto $H$, et al. Differences between right- and leftsided colon cancer in patient characteristics, cancer morphology and histology. J Gastroenterol Hepatol 2008;23:418-423.

25. Heinemann V, Modest DP, von Weikersthal LF, et al. Gender and tumor location as predictors for efficacy: influence on endpoints in first-line treatment with FOLFIRI in combination with cetuximab or bevacizumab in the AIO KRK 0306 (FIRE3) trial [abstract]. J Clin Oncol 2014;32:5s(Suppl):Abstract 3600.

26. American Joint Committee on Cancer. CS Collaborative Stage Data Collection System. Available at: https://cancerstaging.org/cstage/Pages/ default.aspx. Accessed November 6, 2017.

27. National Cancer Institute: SEER Training Modules. General Guidelines for Collaborative Stage. Available at: https://training.seer.cancer.gov/ collaborative/system/. Accessed November 6, 2017.

28. Shankaran V, Ortendahl JD, Purdum AG, et al. Cost-effectiveness of cetuximab as first-line treatment for metastatic colorectal cancer in the United States [online ahead of print September 22, 2015]. Am J Clin Oncol, in press.

29. Tong JH, Lung RW, Sin FM, et al. Characterization of rare transforming KRAS mutations in sporadic colorectal cancer. Cancer Biol Ther 2014;15:768-776

30. Carethers JM, Murali B, Yang B, et al. Influence of race on microsatellite instability and CD8+ T cell infiltration in colon cancer. PLoS One 2014;9:e100461.

31. Troisi RJ, Freedman AN, Devesa SS. Incidence of colorectal carcinoma in the U.S.: an update of trends by gender, race, age, subsite, and stage, 1975 1994. Cancer 1999;85:1670-1676.

32. Petrelli F, Tomasello G, Borgonovo K, et al. Prognostic survival associated with left-sided vs right-sided colon cancer: a systematic review and metaanalysis [published online ahead of print October 27, 2016]. JAMA Oncol, doi: 10.1001/jamaoncol.2016.4227.

33. De Roock W, Claes B, Bernasconi D, et al. Effects of KRAS, BRAF, NRAS, and PIK3CA mutations on the efficacy of cetuximab plus chemotherapy in chemotherapy-refractory metastatic colorectal cancer: a retrospective consortium analysis. Lancet Oncol 2010;11:753-762.

34. Venook AP, Niedzwiecki D, Innocenti F, et al. Impact of primary $\left(1^{\circ}\right)$ tumor location on overall survival (OS) and progression-free survival (PFS) in patients (pts) with metastatic colorectal cancer (mCRC): analysis of CALGB/SWOG 80405 (Alliance) [abstract]. J Clin Oncol 2016;34(Suppl):Abstract 3504.

35. Venook AP, Niedzwiecki D, Lenz HJ, et al. Effect of first-line chemotherapy combined with cetuximab or bevacizumab on overall survival in patients with KRAS wild-type advanced or metastatic colorectal cancer: a randomized clinical trial. JAMA 2017;317:2392-2401.

36. Tejpar S, Stintzing S, Ciardiello F, et al. Prognostic and predictive relevance of primary tumor location in patients with RAS wild-type metastatic colorectal cancer: Retrospective analyses of the CRYSTAL and FIRE-3 trials [published online ahead of print October 10, 2016]. JAMA Oncol, doi: 10.1001/jamaoncol.2016.3797.

37. Kuramochi H, Nakamura A, Nakajima G, et al. PTEN mRNA expression is less pronounced in left- than right-sided colon cancer: a retrospective observational study. BMC Cancer 2016;16:366.

38. Hsu HC, Thiam TK, Lu YJ, et al. Mutations of KRAS/NRAS/BRAF predict cetuximab resistance in metastatic colorectal cancer patients. Oncotarget 2016;7:22257-22270.

39. Loupakis F, Ruzzo A, Cremolini C, et al. KRAS codon 61, 146 and BRAF mutations predict resistance to cetuximab plus irinotecan in KRAS codon 12 and 13 wild-type metastatic colorectal cancer. $\mathrm{Br} \mathrm{J}$ Cancer 2009;101:715-721.

40. Therkildsen C, Bergmann TK, Henrichsen-Schnack T, et al. The predictive value of KRAS, NRAS, BRAF, PIK3CA and PTEN for antiEGFR treatment in metastatic colorectal cancer: a systematic review and meta-analysis. Acta Oncol 2014;53:852-864.

41. Loupakis F, Ruzzo A, Cremolini C, et al. KRAS codon 61,146 and BRAF mutations predict resistance to cetuximab plus irinotecan in KRAS codon 12 and 13 wild-type metastatic colorectal cancer. Br J Cancer 2009;101:715-721.

42. Benson AB III, Venook AP, Cederquist L, et al. NCCN Clinical Practice Guidelines in Oncology: Rectal Cancer. Version 3.2017. Accessed November 6, 2017. To view the most recent version of these guidelines, visit NCCN.org.

43. Benson AB III, Venook AP, Cederquist L, et al. NCCN Clinical Practice Guidelines in Oncology: Colon Cancer. Version 1.2017. Accessed November 6, 2017. To view the most recent version of these guidelines, visit NCCN.org.

44. National Cancer Institute. Surveillance, Epidemiology, and End Results Program. Available at: https://seer.cancer.gov/. Accessed November 6, 2017. 